\title{
Aplicación de la Gestión del Conocimiento Organizacional en la Educación de la Facultad de Ingeniería de la UNJBG
}

\author{
Application on Management of Organizational Knowledge to \\ Educational Process for Engineering - UNJBG
}

\author{
Luis Fortunato Caso Palpa
}

\begin{abstract}
RESUMEN:
Este estudio propone para la Facultad de Ingeniería, un modelo de Gestión del Conocimiento y de Tecnologias de Información, fundamentado en tres aspectos: el capital humano, capital estructural, capital relacional, y considerando como herramienta básica el cambio de cultura organizacional y el monitoreo de la G.C. y las T.I. Todo ello teniendo en consideración que nuestra universidad como toda organización académica, vende $u$ ofrece lo que sabe hacer, y este saber hacer proviene de un grupo de docentes trabajando de forma coordinada con una capacidad intelectual adecuada y Tecnología de Información, obteniendo un producto de calidad, que son los profesionales que aqui se forman. Considerando que la Gestión del Conocimiento y la Tecnologias de la Información nos permitirán crear las condiciones para que el ritmo de enseñanza - aprendizaje en la Facultad y por tanto en la Universidad, sea superior al exigido por el mercado laboral.

Sabiendo que hay conocimientos a gestionar, entonces tiene que haber Rector, Vicerrectores, Decanos y Directores de Escuela quienes serán los principales responsables para el cambio, y serán docentes los participantes de los talleres de trabajo que se organizaran y adoptaran las directivas del cambio con participación de un equipo de trabajo especializado.
\end{abstract}

Palabras clave: Gestión del conocimiento, cultura organizacional, tecnologías de información y comunicación, capital intelectual.

\section{ABSTRACT:}

This study proposes to Engineering Faculty a management model of Knowledge and Information Technologies, based on three aspects: human resources, structure and relational capital as well as using as a basic tool the change of organizational culture and G.C.'s and T.I. All's monitoring. Taking into account the fact that our university like any other academic organization sell or offer what there is available, and this resource of knowledge comes from group of teachers working coordinately with proper intellectual capability and Information Technology, obtaining a high quality product. Those professionals graduated here.

Considering the fact that the management of Knowledge and Information Technologies allow us to provide the conditions for realization of teaching-learning in the Faculty and at the University also, might be higher than the level demanded by the labor market.

As a result of Knowing that there is knowledge to manage, it is necessary to have a Rector, Vice-rectors, Deans and principals who will be responsible for the change and the participants of the workshops will be professors who are to organize and adopt tasks of change with the participation of a group of specialized work.

Key words: Management of knowledge, organizational culture, information technologies and communication, intellectual capital. 


\section{INTRODUCCIÓN}

La Facultad de Ingenieria de nuestra Universidad se encuentra en un entorno complejo en relación a sus puntos fuertes y sus debilidades principales. Sus puntos fuertes de la Facultad son idénticos a las de la universidad, tienen mayor importancia en la actualidad y se caracterizan por que en los últimos años las escuelas de la FAIN se han convertido en una institución educativa de masas, ya que tienen acceso a ella, estudiantes de todas las capas sociales de la región, particularmente los estratos sociales C, D y E. Otra fortaleza es la gran demanda por parte de los egresados de secundaria, motivada por causas variadas. Igualmente, la FAIN ha iniciado procesos de mejoramiento de la investigación con la adquisición de nuevos equipos, así como mejoras pausadas y lentas de modernización de los servicios de Tecnologías de Información y renovación de instalaciones existentes.

Se han analizado algunos puntos críticos de la facultad, con sus implicancias en el proceso de enseñanzaaprendizaje, formación de sus estudiantes y actuación, en las empresas y en la sociedad, habiéndose determinado que uno de los problemas básicos es la oferta docente, que determina un bajo rendimiento en el aspecto académico y en la obtención de grados académicos o títulos. La pobre transmisión de conocimientos dificulta la formación de buenos profesionales que sean aceptados en el mercado de trabajo. Esto es consecuencia del deficiente diseño de los planes curriculares, donde prevalecen generalmente los intereses personales o grupales de la docencia, más no los intereses de la universidad, menos de las empresas y de la sociedad, haciéndose notorio un pobre grado de cultura organizacional. Esta forma de diseño inadecuado del currículo de estudios, no permite la potenciación de las habilidades que poseen los estudiantes y que son esenciales para su acceso al mercado laboral.

La formación profesional en las escuelas de nuestra Facultad carece completamente de actividades formativas que fomenten la iniciativa y creatividad, tanto en aspectos del conocimiento explicito y tácito. Otro problema es la casi inexistente oferta de investigadores de calidad. Esta constatación, además, pone de relieve, el divorcio entre la transmisión de conocimientos y la investigación, poniendo así en entredicho la visión de la universidad: ¿Debe la universidad formar a los profesionales? o ¿Debe priorizar el avance de la ciencia y el conocimiento?

La ausencia de una relación con las empresas y la sociedad es otro problema. Esto es así, porque los convenios, contratos o coordinaciones con las empresas, como empleadoras de sus graduados y demandantes de sus servicios, casi no existen.

El ingreso, promoción y ratificación docente está basada en la subordinación a grupos o la autoridad de turno y con exclusión de aquellos que no se someten a tal conducta. Tal situación se puede verificar en la concesión de incentivos económicos específicamente para aquellos docentes afines que desempeñan cargos administrativos.

Como problema de gestión, es el carácter burocrático de la toma de decisiones y de gestión universitaria a través de la asamblea universitaria, consejo universitario, consejo de facultad y otras instancias directivas, teniendo como integrantes profesores y estudiantes poco preparados para llevar una gestión inteligente en instrucción, formación y administración. Esta clase de gestión, sin vocación, ni formación directiva, hace muy costosa y, a veces, imposible el desarrollo de la institución.

Dado el lugar clave que la Universidad tiene en la vida de la sociedad, se analizan y hacen propuestas de cambio que deben ser aprovechadas por nuestros docentes, administrativos, estudiantes y otros miembros de la comunidad universitaria. Así, la Facultad y sus escuelas profesionales se transformarán de receptores en emisores de conexión, capacitación y servicios a terceros. Se aspira así ofrecer nuevas herramientas para que las escuelas profesionales sigan haciendo el trabajo académico que vienen llevando a cabo desde siempre: educar a los jóvenes de la Región, pero educar bien.

\section{MATERIAL Y MÉTODOS}

\section{MATERIAL}

Se definirán aquí algunos materiales e instrumentos fundamentales para la comprensión del trabajo que deseamos desarrollar:

\section{DATOS}

Los datos describen condiciones, hechos, situaciones o valores, se caracterizan por no contener ninguna información. Un dato puede significar un número, una letra, un signo ortográfico o cualquier símbolo que represente una cantidad, una medida, una palabra o una descripción. Por si mismos los datos no tienen capacidad de comunicar un significado. Para ser útiles, los datos deben convertirse en información y ser verificados o comprobados para ofrecer un conocimiento, idea o conclusión.

Para ser significativos, los datos deben constar de símbolos reconocibles, estar completos y expresar una idea no ambigua. Los símbolos de los datos son reconocibles y pueden ser correctamente interpretados. Muchos tipos diferentes de símbolos comprensibles se usan para transmitir datos.

Decimos que tenemos datos pertinentes o relevantes cuando pueden ser utilizados para responder a preguntas propuestas. Solo los hechos relacionados con las necesidades de información son pertinentes. Así la organización selecciona hechos entre sucesos y entidades particulares para satisfacer sus necesidades de información.

\section{INFORMACIÓN}

La información no es un dato. Es más bien una colección de hechos significativos y pertinentes, para el organismo u organización que los percibe. La definición de información es la siguiente: Información es un conjunto de datos significativos y pertinentes que describen sucesos o entidades.

Las personas generan datos que luego transforman en información, posteriormente a través del análisis y el uso productivo de la misma, la transforman en conocimiento. La información es un mensaje con un contenido determinado emitido por una persona hacia otra y, representa un papel primordial en el proceso de la comunicación. La información tiene significado para 
quien la recibe, por eso, los seres humanos siempre han tenido la necesidad de cambiar entre sí información que luego transforman en acciones. La información es, entonces, conocimientos basados en los datos a los cuales, mediante un procesamiento, se les ha dado significado, propósito y utilidad.

\section{ELCONOCIMIENTO}

El Conocimiento es un conjunto integrado de información, reglas, interpretaciones y conexiones puestas dentro de un contexto y de una experiencia, que ha sucedido dentro de una organización, bien de una forma general o personal. El conocimiento sólo puede residir dentro de una persona determinada que lo interioriza racional o irracionalmente.

El conocimiento es personal, en el sentido de que se origina y reside en las personas, que lo asimilan como resultado de su propia experiencia (es decir, de su propio "hacer", ya sea físico o intelectual) y lo incorporan a su acervo personal estando "convencidas" de su significado e implicaciones, articulándolo como un todo organizado que da estructura y significado a sus distintas "piezas".

Su utilización, puede repetirse sin que el conocimiento "se consuma" como ocurre con otros bienes físicos, permite "entender" los fenómenos que las personas perciben (cada una "a su manera", de acuerdo precisamente con lo que su conocimiento implica en un momento determinado), y también "evaluarlos", en el sentido de juzgar la bondad o conveniencia de los mismos para cada una en cada momento.

\section{GESTIÓN DELCONOCIMIENTO}

Su función consiste en planificar, poner en marcha, operar y controlar todas las actividades y programas relacionados con el conocimiento, que requiere una gestión eficaz del capital intelectual. Gestión del Conocimiento es un proceso tanto cultural y tecnológico que busca captar y transformar el conocimiento individual para sistematizarlo y convertirlo en información valiosa de acceso colectivo.

La Gestión del Conocimiento es, en definitiva, la gestión de los activos intangibles que generan valor para la organización. La mayoría de estos intangibles tienen que ver con procesos relacionados de una u otra forma con la captación, estructuración y transmisión de conocimiento. "La inteligencia se convierte en un capital cuando se crea un orden útil a partir de la capacidad intelectual, es decir cuando se le da una forma coherente"... "cuando se aprende de manera tal que se pueda describir, difundir y explotar, y cuando se puede emplear para hacer algo que no se podría realizar ni siquiera desparramado como monedas en una alcantarilla. El capital intelectual es conocimiento útil envasado."

\section{LACULTURAORGANIZACIONAL}

La cultura organizacional da las pautas acerca de cómo las personas deben conducirse en ésta. En muchas ocasiones la cultura es tan evidente que se puede ver que la conducta de la gente cambia en el momento en que traspasa las puertas de la empresa. La cultura es el vínculo social que ayuda a mantener unida a la organización al proporcionarle normas adecuadas de cómo deben comportarse y expresarse los empleados.

El mal conocimiento de la cultura puede ser causa de malos entendidos y malas interpretaciones dentro de las organizaciones en el momento en que pierde su capacidad de coordinación y de integración. La cultura organizacional es la médula de la organización que está presente en todas las funciones y acciones que realizan todos sus miembros. Es importante conocer el tipo de cultura de una organización, porque los valores y las normas van a influir en los comportamientos de los individuos.

Cuando la cultura de una organización está bien sustentada por sus valores, se busca que todos los integrantes de ésta desarrollen una identificación con sus propósitos estratégicos y desplieguen conductas de desarrollo y automotivación. La capacitación continua del personal de la empresa es un elemento fundamental para dar apoyo a la creación de un programa orientado al fortalecimiento del sentido de compromiso del personal hacia la empresa, cambiar actitudes y fomentar la comunicación, comprensión e integración de las personas.

\section{CAPITAL INTELECTUAL}

El capital intelectual es la suma de lo que saben todos en la institución y que estructurado, le proporciona una ventaja competitiva en el mercado al que se orienta. Este conjunto de conocimientos debe ser renovable por que los cambios a los que se enfrenta la institución son constantes, al mismo tiempo debe ser éste tipo de capital democrático ya que debe haber participación por parte de los trabajadores en la planeación de las actividades y en la distribución de los recursos para lograr alcanzar los objetivos de la organización; asimismo para aprovechar el capital intelectual se tiene que estructurar, capitalizar y sistematizar la información.

\section{METODOLOGÍA}

a. Análisis de Gestión del Conocimiento Organizacional en el proceso educativo de la FAINUNJBG

Para elevar la competitividad de la Facultad, el mayor desafio que enfrenta es la transformación de la calidad educativa. Las trasformación educativa, al igual que las trasformación productiva, en una región como la nuestra está determinada por un entorno de globalización e internacionalización de las empresas y por las estructuras productivas. 
El actual desarrollo Tecnológico-Productivo del Perú, desde la década del 90 es objeto de transformación dependiente, recurriendo a la importación y consumo de equipos, materiales e insumos. Esta política en nuestro pais en vías de desarrollo, no ha resuelto la diferencia Tecnológica ni la productiva con respecto al desarrollo económico de los países industrializados, porque no ha creado ninguna estructura productiva con tecnologia propia ni una base científica, tecnológica y de capital intelectual.

Nuestra Facultad, sigue el mismo camino de adaptación mecánica al proceso de liberalización económica y corre el peligro de convertirse en una institución educativa que refuerza la división y desigualdad social, desintegración, el desequilibrio urbano-rural. Por ello la transformación educativa de la FAIN-UNJBG es un proceso clave para responder a las nuevas necesidades de la sociedad del conocimiento y detener los procesos de desintegración social.

Nuestra Facultad, debe generar una educación que prepare para la vida al nuevo profesional en un proceso productivo cambiante, menos jerárquico, y basado en organizaciones integradas por redes, con carreras que no serán lineales y cuya fronteras no serán las de un país sino las del mundo, donde se necesitará mas iniciativa que docilidad, mas creatividad que mandatos. Por consiguiente, la educación del futuro debe tender a generar profesionales con capacidad de meditación, un pensamiento sistémico e interrelacionado, con habilidad de experimentación, capacidad de colaboración, trabajo en equipo e interacción con los pares. En suma, una educación que cree una mente dinámica innovadora y con sólidos principios éticos y morales.

Para hacer realidad esto, se debe dotar a la FAIN y a la universidad de una cultura organizativa y aprendizaje organizacional como elementos básicos de un sistema de información, con un modelo de gestión de conocimiento que permita producir profesionales competitivos.

\section{Construcción de una cultura innovadora en la FAIN-UNJBG}

La cultura de innovación es un conjunto de presunciones, valores y comportamientos que permitirán a los docentes llevar a cabo innovaciones sin resistencias. Se plantea este paradigma porque en la actualidad la facultad está destinada a cambiar si quiere continuar siendo la institución que transmita conocimientos para el desarrollo de la sociedad. Debe tener una cultura organizativa predispuesta al cambio. Sin embargo, para poder llegar a una cultura de cambio, es necesario adoptar estrategias que contribuyan a recrear la cultura existente para convertirla en una cultura en la que los valores y conductas predominantes sean las de aceptación al cambio, interés para mejorar, etc. características de una cultura organizacional innovadora.
La capacidad académico-administrativa de nuestra facultad debe responder a tres grandes retos: Primero, la atención a la diversidad socio - económica de

estudiantes, la necesidad del aprendizaje continuo a lo largo de la vida, la capacidad de ofrecer una formación profesional adecuada para el crecimiento económico, una apuesta más firme en tecnología, ciencia e ingeniería.

Segundo, la agenda de la calidad académica guarda relación con los resultados que puede conseguir la facultad, que se traduce en la capacidad de medir estos resultados, por ejemplo calculando el número de graduados que finalizan los estudios en el tiempo estimado; los costos, cuotas o retorno de la inversión, el ofrecimiento de créditos y carreras a tiempo parcial, diplomados, estudios de post grado, etc. La universidad debe cambiar internamente para dejar de ser una institución compleja y muy difícil de gestionar.

En tercer lugar, la restructuración de las fuentes de financiación por RDR; la reorganización curricular de facultades, escuelas, departamentos; el establecimiento de nuevas alianzas con otras entidades públicas y privadas, los vínculos con los centros de tecnología e investigación de otras instituciones, etc., suponen otro tipo de cambios a los que debemos enfrentar.

El cambio de paradigma de la docencia hacia una mayor autonomía del estudiante que no ha sido efectivo; sobretodo cuando ha mostrado la inexistencia de una planificación de la carrera profesional con los incentivos necesarios para cada función y ha presenciado una baja identificación de los estudiantes con los estudios.

\section{b. Diagnóstico de la cultura organizativa de la FAIN y la UNJBG}

El modelo, desarrollado para hacer el diagnostico de la cultura organizativa se experimenta y complementa mediante un estudio que permite investigar el fenómeno en su contexto real. Del mismo modo, la utilización de múltiples casos proporciona al estudio final una mayor vitalidad. Describimos la cultura organizacional a través de:

a. La percepción de la facultad manifestada por sus integrantes sobre un cuestionario de preguntas;

b. La observación del comportamiento docente, valores y fortalezas que la facultad hace gala;

c. El análisis de documentos institucionales como los Estatutos, reglamentos, guías, textos, página web.

d. Las percepciones acerca de las autoridades responsables de diferente rango.

$\mathrm{Y}$ todo ello a través de los siguientes instrumentos y estrategias: 
Tabla $\mathbf{N}^{\circ} 01$. Estrategias e instrumentos para la identificación de la Cultura Organizacional.

\begin{tabular}{|c|c|c|}
\hline $\begin{array}{c}\text { Tipo de } \\
\text { Recolector de } \\
\text { Dato }\end{array}$ & Dirigido a & objetiva \\
\hline Observaciones & $\begin{array}{l}\text { Simbolos } \\
\text { representativos } \\
\text { Cosiumbres, valores } \\
\text { emblema }\end{array}$ & $\begin{array}{l}\text { Existenciao node } \\
\text { representaciones } \\
\text { costumbres } \\
\text { aceptables }\end{array}$ \\
\hline $\begin{array}{l}\text { Analis is de } \\
\text { documentos }\end{array}$ & $\begin{array}{l}\text { Documentosde las } \\
\text { escuelus y to que ha } \\
\text { generado la } \\
\text { facultad: estatutos. } \\
\text { normas, guias } \\
\text { académicas... }\end{array}$ & $\begin{array}{l}\text { Comose manifiesta } \\
\text { ta faculiad sobre } \\
\text { taspregun ias y et } \\
\text { andisis de los } \\
\text { dacumentos que la } \\
\text { representan }\end{array}$ \\
\hline Enlrevisias & $\begin{array}{l}\text { Responsables } \\
\text { académlcos y } \\
\text { adm in is trativos }\end{array}$ & $\begin{array}{l}\text { Percepción } \\
\text { callficada sobre la } \\
\text { validez de la } \\
\text { información } \\
\text { obten ida }\end{array}$ \\
\hline Cuestionatio & $\begin{array}{l}\text { Docentes, } \\
\text { Adm in is trativos } \\
\text { Estudiantes }\end{array}$ & $\begin{array}{l}\text { Percepción sobre } \\
\text { lasrespuestas del } \\
\text { cuestionario y } \\
\text { evaluactón }\end{array}$ \\
\hline
\end{tabular}

Tras un estudio preliminar, en el que se realiza un análisis comparativo de cuestionarios similares, se definen las dimensiones centrales de la cultura organizacional universitaria y se delimitan particularidades de la población a estudiar, se genera un instrumento para identificar dicha cultura: Cuestionario de Identificación de la Cultura Organizacional Universitaria. El cuestionario no sólo pretende identificar la cultura actual, sino que permite compararla con la cultura deseada, facilitando así la gestión de procesos de cambio organizativo.

Tabla $\mathbf{N}^{\circ} \mathbf{0 2}$. Estructura del cuestionario

\begin{tabular}{|c|c|c|}
\hline Dimensión & Contenido & $\begin{array}{c}\text { No. } \\
\text { itcm s }\end{array}$ \\
\hline Funciones & $\begin{array}{l}\text { Dualidad: Docencia - Investigación } \\
\text { Universidad - Sociedad } \\
\text { Desarrollo territorial }\end{array}$ & \\
\hline $\begin{array}{c}\text { Gob ierno y } \\
\text { Gestion }\end{array}$ & $\begin{array}{l}\text { Nombramionto del Rector } \\
\text { Cargos de gestión acad ćmica } \\
\text { Órganos degestión } \\
\text { Autonom ia de gobiemo }\end{array}$ & \\
\hline F inanciación & $\begin{array}{l}\text { Modelo de financiación } \\
\text { A signación de presupuesto publico } \\
\text { A portación financierade los } \\
\text { usuatios }\end{array}$ & \\
\hline Inves tiga ción & $\begin{array}{l}\text { Criterios de evaluación y } \\
\text { reconocimiento } \\
\text { Criterio de motivación } \\
\text { Criterio de creación de grupos de } \\
\text { investigación }\end{array}$ & \\
\hline $\begin{array}{l}\text { Perfildel } \\
\text { profesar }\end{array}$ & $\begin{array}{l}\text { Funciones del profesor } \\
\text { universitario } \\
V \text { ision de la disciplina } \\
V \text { isión de los alum nos } \\
T \text { rabajo en equipo en el } \\
\text { devartamento }\end{array}$ & \\
\hline M elod ologia & $\begin{array}{l}\text { Transmisión de conocim ientos } \\
\text { Actual ización de la materia } \\
\text { Introducción de actividades } \\
\text { Evaluación de los estudiantes }\end{array}$ & \\
\hline $\begin{array}{l}\text { Evaluación e } \\
\text { innovación }\end{array}$ & $\begin{array}{l}\text { Metodos de formación } \\
\text { Función como estamento de la } \\
\text { universidad } \\
\text { Interacción con los docen tes y } \\
\text { estudiantes } \\
\text { Evaluación institucional } \\
\text { Evaluación del docente }\end{array}$ & \\
\hline $\mathrm{TIC}$ & $\begin{array}{l}\text { Gestión Admin istrativa } \\
\text { Docencia e investigación } \\
\text { Infracstructura }\end{array}$ & \\
\hline $\begin{array}{l}\text { Relaciones de la } \\
\text { Facultad con su } \\
\text { entomo }\end{array}$ & $\begin{array}{l}\text { Convenios con empresas } \\
\text { R claciones intemacionales con } \\
\text { oiras instituciones } \\
\text { Practicas } \\
\text { Estudios de Dost grado }\end{array}$ & \\
\hline
\end{tabular}

El cuestionario consta de más de 100 preguntas, cada una de las cuales pretende conocer la percepción del profesorado universitario frente a la cultura universitaria actual (escala A) y la cultura universitaria esperada (escala B) de la institución en la que trabaja.

\section{RESULTADOS}

1. Propuestas para mejorar la gestión del conocimiento en la FAIN-UNJBG

Si la Facultad no logra mejorar sus procedimientos de administración, identificación y control del conocimiento de susdocentes, estudiantes y administrativos, puede llegar a serios problemas en relación a la ventaja competitiva con el resto de universidades y sobre todo desperdiciar una de las mejores fuentes para crear y aumentar valor. Con esto, se pierden ventajas competitivas, al dejar de lado un activo muy importante: El Capital Intelectual.

El conocimiento es uno de los recursos más importantes de la institución, capturarlo estratégicamente, es un proceso que involucra el compromiso de cada integrante y requiere un nivel de prioridad importante.

En el mundo competitivo en el que estamos viviendo, los activos mas valiosos ya no son los tangibles tales como la maquinaria, ; los equipos, las herramientas, los edificios, las instalaciones, etc., sino los intangibles que tienen su nacimiento en los conocimientos, las actitudes de sus integrantes, las habilidades, etc. Aquí se propone una sencilla clasificación de los activos intangibles:

a. Competencia individual: es la educación, el "knowhow", los valores y actitudes de cada uno de los integrantes, las habilidades específicas y los conocimientos. Este tipo de activo, no es propiedad de la Universidad, ya que la misma contrata el uso de ellos como docentes. Estos, al no formar parte ya de la Universidad, se llevan consigo estos activos. Estos activos también se conocen como "Capital humano"

b. Estructura interna: son los métodos y procedimientos de internos de trabajo, las bases de datos de sus procesos administrativos y académicos, la producción intelectual a través de la $\mathrm{I}+\mathrm{D}$ (investigación y desarrollo) y la cultura de la Universidad. En este caso, estos activos si son propiedad de la Universidad y algunos de ellos se pueden proteger (investigaciones, textos, patentes, etc.)

c. Estructura externa: están relacionados a las empresas que absorben a los profesionales que salen de nuestra Universidad, las relaciones con instituciones que mediante convenios se firman los acuerdos de cooperación, tecnologías, alianzas estratégicas, a las marcas comerciales. Estos activos son propiedad de la Universidad y también pueden protegerse legalmente.

\section{Propuesta de innovación de la FAIN - UNJBG}

La universidad y por consiguiente nuestra Facultad están cambiando sin ser el motor que impulsa el proceso de innovación de la sociedad, sino que actúan, como ejecutores de cambios que han sido impuestos a nivel político nacional o bien por influencia de la competencia interna entre universidades $u$ otras presiones externas. Esto no niega la existencia de una habilidad interna de algunos docentes para fomentar e implementar procesos 
innovadores. Pero por lo general, la innovación esta ausente. Esto nos obliga que nuestros objetivos deben estar orientados a impulsar la innovación de nuestros objetivos, estructuras, servicios y procesos. Sin embargo, el final de este ciclo de innovación está lejos de terminar y es muy probable que surjan en un futuro cercano nuevos factores de cambio.

La tarea más importante e inmediata para la FAIN y UNJBG es construir una cultura que aprecie y apoye el cambio en todos los niveles organizativos. Deben crearse, articularse y adoptarse nuevas estrategias institucionales que permitan a la universidad sobrevivir y prosperar en este nuevo mercado educativo.

El análisis de la actividad innovadora en la FAIN revela cuatro elementos básicos que deben implementarse:

\section{Un gran equipo de gestión de cultura académica}

Se debe implementar un equipo directivo con mucha autoridad y un gran sentido de autonomía. Pueden ser las autoridades principales o de cualquier nivel intermedio. La clave para un buen equipo reside en la adaptabilidad combinada con una habilidad administrativa de fusionar nuevos valores de gestión con los valores académicos tradicionales de manera que todos los niveles trabajen para mejorar la cultura académica.

\section{Desarrollar vinculos con el entorno empresarial}

El establecimiento de vínculos entre la universidad y las empresas que absorben nuestros profesionales para generar y promocionar el conocimiento permitirá un beneficio mutuo. El resultado de establecer estas relaciones es la expansión de la transferencia de conocimiento, el contacto industrial, el desarrollo de la propiedad intelectual, el aprendizaje continuo y contactos entre los participantes.

\section{Una facultad consciente y motivada}

Se refiere a todas las funciones académicas de la facultad. La mayoria de los valores tradicionales buenos y/o malos están profundamente arraigados en los docentes. Para que nuestra facultad se encuentre plenamente identificada con el proceso innovador, cada docente debe aceptarlo y involucrarse en el proceso. Los docentes de un departamento mantienen los valores y prácticas académicas tradicionales mientras simultáneamente integra nuevas prácticas de gestión, tecnología y de mercado.

\section{Cultura académica innovadora e inteligente}

El objetivo no es crear una cultura académica nueva que remplace a la anterior. Uno de los retos de la innovación es hacer compatible los cambios con la tradición y el prestigio de la universidad dejando de lado algunas innovaciones que pueden poner en peligro la identidad de la facultad. Aun cuando lleven a cabo procesos de innovación interna, la facultad debe seguir siendo ella misma.
Los primeros tres elementos se combinan para crear una cultura que involucra cambio y mantiene los valores fundamentales de la institución. Mientras el espíritu emprendedor $\mathrm{e}$ innovador puede empezar por un departamento académico, una institución emprendedora facilita el desarrollo de una cultura que engloba la idea a un nivel más institucional.También se señalan algunos factores importantes para avanzar hacia la construcción de una facultad innovadora:

\begin{tabular}{|c|c|}
\hline $\begin{array}{c}\text { Factores } \\
\text { Importantes }\end{array}$ & Propuestas de ca mbio \\
\hline Visión, Misión & $\begin{array}{l}\text { Para adaptarse, la facultad necesita } \\
\text { que la Universidad defina } \\
\text { claramente su misión y visión. }\end{array}$ \\
\hline Cultura & $\begin{array}{l}\text { Una cultura organizacional desde } \\
\text { el punto de vista académico } \\
\text { aumenta la capacidad de } \\
\text { adaptación de la facultad }\end{array}$ \\
\hline Estructura & $\begin{array}{l}\text { La estructura organizacional debe } \\
\text { "prepararse" para aumentar su } \\
\text { adaptación y ser diferente de las } \\
\text { demás. }\end{array}$ \\
\hline Entorno & $\begin{array}{l}\text { La adaptación de la facultad es } \\
\text { mas por las demandas del entorno } \\
\text { y en momentos de crisis. Es una } \\
\text { oportunidad para la facultad. }\end{array}$ \\
\hline $\begin{array}{c}\text { Especialización } \\
\text { acad émica }\end{array}$ & $\begin{array}{l}\text { Una profesionalización con } \\
\text { aprendizaje continuo ayuda a la } \\
\text { adaptación. }\end{array}$ \\
\hline $\begin{array}{c}\text { Gobiemo } \\
\text { Institucional }\end{array}$ & $\begin{array}{l}\text { Un gobierno de participación con } \\
\text { menos sectarismo es necesario } \\
\text { para implementar cambios de } \\
\text { adaptación. }\end{array}$ \\
\hline Liderazgo & $\begin{array}{l}\text { El compromiso del lider es } \\
\text { esencial para la adaptación }\end{array}$ \\
\hline
\end{tabular}

\section{Impacto de la tecnologia en la cultura innovadora}

En la sociedad de la información y el conocimiento la facultad está destinada a crear, criticar y difundir el conocimiento haciendo uso de los recursos tecnológicos que complementan a los métodos y recursos pedagógicos para llegar a más estudiantes. Los entornos virtuales de aprendizaje y los cursos a distancia forman parte del espacio de aprendizaje universitario; los sistemas de gestión de la información y los sistemas de gestión de tareas del profesorado están uniendo esfuerzos para mejorar la comprensión de la actividad universitaria y regularla.

Es necesario que la facultad adopte la filosofia del «tiempo lento» para poder comprometerse de manera profunda con la sociedad. Para estos tiempos de cambio es necesario:

- Entender que la facultad es una comunidad de académicos y debe ser reflejo y conciencia crítica de la sociedad así como promotora de un intelecto democrático positivo.

- Compartir el conocimiento y realizar investigación conjunta. Preparar a los estudiantes para el reto intelectual, la curiosidad y la satisfacción. 
La facultad es parte de la universidad que tiene una estructura académico-administrativa «débilmente acoplada» donde existen anarquías organizadas: por los bajos mecanismos de control, por la mal entendida autonomía universitaria y una pobre descentralización en cuestiones docentes, la investigación que interesa, etc. Las consecuencias de tener esta organización «débilmente acoplada» son:

a) Tener un poder no jerarquizado, informal $y$ diseminado de manera desigual a través de la organización como jefes de departamentos, directores de escuela, decanos de facultades, investigadores, profesores socialmente visibles, etc. Un funcionamiento caótico y la autocracia como estrategia para construir imperios con mecanismos perversos de socialización

b) Si bien el conocimiento se basa en el desarrollo y en las decisiones, la reducida cultura organizacional de sus miembros y la intensa actividad proselitista evitan el desarrollo institucional.

c) La descentralización puede considerarse un beneficio. Pero significa tener instituciones «invisibles» con límites imprecisos. Como consecuencia se generan dinámicas micro-feudos dificiles de gestionar.

d) Las redes sociales de grupos que se forman y defienden intereses ajenos a la institución no permiten democratizar el manejo administrativo, con la consecuencia negativa de la creación de defensas corporativas y poco poder a los estudiantes oprofesores jóvenes.

A nivel de docentes, existen algunas actitudes que pueden cambiarse con el fin de mejorarla:

- La forma en que los departamentos asignan la carga horaria de los profesores.

- La forma en que se promueven espacios para compartir metodologías de enseñanza o proyectos de investigación.

- La distribución de espacios físicos para los docentes.

- Las normas académicas de trabajo de los profesores por parte de las escuelas o facultades.

- La organización de la capacitación y la orientación a los nuevos profesores.

Además de promover un estilo de liderazgo transformador, la existencia de buenas prácticas organizativas también es sinónimo de cambio de cultura. Algunas de ellas son: la socialización de profesores, la importancia de los acontecimientos, la importancia de las redes sociales de comunicación con diferentes medios y recursos, la importancia de espacios y tiempos como expresiones simbólicas de la cultura, el apoyo a las actividades interdisciplinarias y el reflejo de prácticas éticas en la organización.

\section{CONCLUSIONES Y RECOMENDACIONES}

\section{CONCLUSIONES}

- Los cambios sociales, económicos, tecnológicos $\mathrm{y}$ culturales afectan a la universidad, a sus sistemas de enseñanza, a la organización de la investigación, a la docencia y a la gestiónacadémica, involucrando una nueva cultura organizacional que nos hace falta para sensibilizar y preparar al personal docente,no docente $\mathrm{y}$ los estudiantes.

- La relación de la Facultad con la empresa y sociedad debe aumentar, vinculándose a través de oficinas de Investigación y Transferencia Tecnológicas promoviendo relaciones estrechas y ejerciendo funciones de dinamización cultural en y para la sociedad.

- La mayoria de personas encuestadas opinan que la elección del rector debe expresar el sentir democrático de la Universidad y manifiestan no estar de acuerdo con que esta elección corresponda a la asamblea universitaria; opinión que comparten sectores profesionales al solicitar una elección universal e interna.

1 Muchos encuestados opinan que los cargos directivos de la universidad deberían ser más profesionales y que el problema se podría atenuar si el profesorado tuviera formación administrativa adecuada al acceder a cargos de gestión.

\ La función actual del docente universitario es ejercer de mediador y facilitador en el aprendizaje de los estudiantes. La función docente se debe concentrar más en el desarrollo de una serie de competencias académicas y profesionales, basadas en la reflexión y contribuyan a la transformación de la sociedad y al desarrollo crítico de sus estudiantes, y se concentre menos en la transmisión de conocimiento tradicional del profesor.

- El profesor es un elemento clave en la formación del estudiante, pero no es el único. Existen otros recursos que el estudiante tiene a su disposición y el profesor debe organizarlos para que se gestione el conocimiento de manera individualizada para conseguir objetivos comunes y propios del aprendizaje. Deben entender que el conocimiento de una asignatura no se transmite unidireccionalmente, sino que debe dar margen de maniobra a los estudiantes para que cada uno llegue a sus propias metas.La formación del estudiante debe considerar la educación cultural y humanista como la especialización en un área de conocimiento.

La actual metodología de enseñanza tipo expositiva no debe ser la estrategia prioritaria ni única en la docencia universitaria, se requieren estrategias innovadoras más participativas y reflexivas, además de un apoyo explícito mediante tutorías presenciales o virtuales. Esta innovación pasa por un cambio de 
concepciones de los docentes y una formación, pero también por un cambio de actitud en algunos estudiantes.

$4 \quad$ La actualización del profesor es imprescindible y necesaria pero también se apunta la idea de que hace falta un conocimiento más básico y amplio de la materia por parte del docente. La forma en que debe darse esta actualización es a través de la lectura de documentos, a partir de la reflexión sobre la experiencia y también mediante la investigación.

$\Delta \quad$ La FAIN sigue atrapada en el desfinanciamiento de sus actividades, tanto por parte del estado, así como producto de sus actividades de generación de fondos RDR, exceptuando los recursos de canon minero destinado para proyectos específicos. Con la estructura de financiamiento actual que posee la universidad es incierta la posibilidad de aumentar la calidad de la docencia y de la investigación.

\ Las empresas que gestionan el conocimiento, maximizan sus beneficios por encima de aquellas organizaciones que no se interesan por generar, transferir y utilizar eficientemente y efectivamente su conocimiento. En la presente investigación, se analizó exclusivamente dos fases de la gestión del conocimiento: la generación y la transferencia del conocimiento. Se han dejado pendiente de estudio y la utilización del conocimiento, ya que se deseaba observar los procesos que cuentan con un mayor componente tácito. El incluir la fase de utilización en un estudio posterior habría de traer como resultado una visión integral del proceso de la gestión del conocimiento, al estudiar la forma en que éste se utiliza, como se crea y se transfiere.

\ Considerar este estudio desde una perspectiva formativa aportará, sin dudas evidencia sobre una mejor forma de gestionar el conocimiento. Variables como compromiso y confianza en la organización, determinantes motivacionales para generar y transferir el conocimiento, pueden ser muy relevantes.

\ El mejoramiento continúo de la calidad académica del personal docente a nivel de actualización (Post Grado, Post Doctoral y Training especializado) se va a incrementar si mejora la cultura organizacional de la universidad.

- Optimización de la gestión universitaria en los aspectos administrativos, y procedimientos académicos en los próximos años.

\ Promoción y establecimiento de la formación profesional con sólidos valores ético-morales en los próximos años.

\ Aprovechamiento óptimo de los recursos económico - financieros en la Gestión del Conocimiento elevando su rendimiento durante los próximos años.

\ Creación de unidades de producción altamente rentables como fuente de recursos propios de la universidad con un alto crecimiento anual y reducción de los niveles de desempleo del egresado de la Universidad.

\section{RECOMENDACIONES}

\ Un sistema de gestión del conocimiento que se implemente debería ser actualizado continuamente, al menos una vez por año, para que los estamentos recurrentes no pierdan interés.

- Sería muy conveniente buscar vínculos con otras universidades nacionales que quieran compartir sus experiencias al respecto.

\ Se deberá contar con personal de capacitación, para la instrucción y entrenamiento de los profesores, a fin de obtener los resultados esperados.

- Actividades propuestas para las escuelas profesionales: Instituto de Investigación de la Facultad de Ingenieria

\section{REFERENCIAS BIBLIOGRÁFICAS}

[1] Viedma, J.M. La Gestión del Conocimiento y el Capital Intelectual. (1998). 1

[2] David A. Klein y Laurence Prusak, "Characterizing intellectual capital", pág. 10. Marzo 1994.

[3] Thomas A. Stewart, "La nueva riqueza de las organizaciones: El capital intelectual, edición 1998. Granica.

[4] Nevado, D. \& López, V. (2002). El capital intelectual: Valoración y medición.

[5] Sullivan, P. (2001). Rentabilizar el capital intelectual. Paidós empresa.

[6] Collison, CH. \&Parcell G. (2003). La gestión del conocimiento. Lecciones prácticas de una empresa lider. Paidos Empresa. España.

\section{Referencia Electrónica:}

- http://www.microsoft.com/spain/technet/recursos/p resentaciones/gestion/Gates-ES_archivos/frame.htm

- www.managementweb.com, portal sobre gestión de negocios.

- http://www.floridapartnership.usf.edu

- http://knowab.co.uk/wbwload.html

- http://www.jucs.org/jucs_3_8/information_technol ogy_for_knowledge/paper.html

- $\overline{\mathrm{h}} \mathrm{ttp} / \overline{/} / \mathrm{hsb}$.baylor.edu/ramsower/ais.ac.96/papers/R

AVEN.htm

- http://www.cba.neu.edu/ - mzack/articles/fourprob/f ourprob.htm

- http://www.cba.neu.edu/ mzack/articles/kstrat/kstr at.htm

- http://www.cba.neu.edu/ mzack/articles/socnet/soc net.htm

\section{Correspondencia:}

Luis Fortunato Caso Palpa

Ciudad Universitaria Fundo "Los Granados"

Av. Miraflores s/n Tacna. Perú.

luis_caso@hotmail.com 